\title{
The impact of geomagnetic substorms on GPS receiver performance
}

\author{
S. Skone and M. de Jong \\ Department of Geomatics Engineering, University of Calgary, 2500 University Dr. NW, Calgary, Alberta, Canada T2N 1N4
}

(Received January 13, 2000; Revised August 12, 2000; Accepted September 25, 2000)

\begin{abstract}
During the current period of solar maximum, there is concern within the GPS community regarding GPS receiver performance during periods of intense geomagnetic substorms. Such storms are common in the high latitude auroral region, and are associated with small-scale scintillation effects, which can cause receiver tracking errors and loss of phase lock. The auroral oval can extend many degrees equatorward under active ionospheric conditions, with an impact on precise positioning applications in Canada, the United States and Northern Europe. In this paper, a study of receiver tracking performance is conducted during periods of auroral substorm activity. Dual frequency observations are obtained using codeless and semicodeless GPS receivers (Trimble 4000SSi, NovAtel MiLLennium and Ashtech Z-12), and performance comparisons are established and interpreted with respect to GPS availability at solar maximum and the years beyond.
\end{abstract}

\section{Introduction}

The ionosphere is a dispersive medium, in which RF signals are refracted by an amount dependent on the given signal frequency and ionospheric electron density. In regions of small-scale irregularities in electron density, rapid random phase variations can be produced by phase irregularities in the emerging wavefront (cf. Hargreaves, 1992). These are referred to as phase scintillations. Diffraction of the signal (interference across the wavefront) also leads to variations in signal amplitude - referred to as amplitude scintillation (or amplitude fading, for degradations in signal strength). These effects are strongest in the equatorial $\left( \pm 10^{\circ}-20^{\circ}\right.$ geomagnetic latitude), auroral $\left(65^{\circ}-75^{\circ}\right.$ geomagnetic latitude) and polar cap $\left(>75^{\circ}\right.$ geomagnetic latitude) regions. This paper focuses on high latitude auroral scintillations, which are associated with an enhanced solar wind-magnetosphere interaction. A brief description of this phenomenon follows.

Auroral irregularities result from a precipitation of energetic electrons along terrestrial magnetic field lines into the high latitude ionosphere. These electrons are energized through complex interactions between the solar wind and the Earth's magnetic field, resulting in optical and UV emissions commonly known as the aurora borealis/australis. This phenomenon characterizes the geomagnetic or magnetospheric substorm, where associated irregularities in electron density (at altitudes of 100-500 km) lead to scintillation effects (cf. Aarons, 1982). Auroral intensifications, during a substorm event, have times scales on the order of 15 minutes and, for intense events, multiple intensifications can take place over a period of hours (Rostoker, 1991). The auroral oval can expand several degrees equatorward during such events (i.e. over Northern Europe, Northern United States-see Fig. 2), and the magnitude and frequency of substorms are functions

Copy right (C) The Society of Geomagnetism and Earth, Planetary and Space Sciences (SGEPSS); The Seismological Society of Japan; The Volcanological Society of Japan; The Geodetic Society of Japan; The Japanese Society for Planetary Sciences. of the solar cycle.

GPS receiver tracking performance can be degraded in the presence of scintillation effects. Rapid phase variations cause a Doppler shift in the GPS signal, which may exceed the bandwidth of the phase lock loop (PLL), resulting in a loss of phase lock (Leick, 1995). Additionally, amplitude fades can cause the signal-to-noise-ratio (SNR) to drop below receiver threshold, resulting in loss of code lock. These effects have a larger impact on tracking loops employing codeless and semicodeless technologies, versus full code correlation. In particular, codeless and semicodeless tracking loops experience losses of 27-30 dB and 14-17 (Leick, 1995), respectively, with respect to full code correlation, and are therefore more susceptible to the effects of amplitude fading. The L2 PLL also employs a narrower bandwidth $(\approx 1 \mathrm{~Hz}$, compared with $\approx 15 \mathrm{HZ}$ for L1) to eliminate excess noise, and is more susceptible to phase scintillations. These effects are therefore a significant concern for users who require dual frequency data (i.e. for estimation of ionospheric effects, or resolution of widelane ambiguities).

Several researchers have studied the impact of scintillations on GPS receiver performance. Nichols et al. (1999) investigated high latitude scintillation effects during an intense substorm event in August 1998. They observed degraded L2 phase tracking performance for periods of up to one hour, with L2 data dropouts primarily in the range 40$200 \mathrm{~s}$. These results were derived using a codeless receiver. In contrast, Clynch and Aarons (1996) observed no losses of lock for TurboRogue and Rogue receivers operating at high auroral latitudes during magnetic storm activity in 1995. For an earlier event in 1992, Clynch and Henry (1994) observed simultaneous loss of lock events on two-three satellites during one 20-minute period of intense scintillation, using an older version Ashtech P-12 receiver located near the South Pole. An investigation of NovAtel Millennium receiver performance was recently conducted by Knight et al. (1999), for 
an array of eight GPS receivers in the equatorial region. Periods were observed during which L2 phase observations were corrupted up to $27 \%$ of the time, and loss of L2 code lock was often observed. L1 tracking performance was degraded to a lesser extent.

The results of such studies depend not only on the magnitude of scintillation activity observed, but also on the receiver tracking capabilities - which can vary widely between manufacturers and models. In order to fully assess the impact of ionospheric activity on GPS signal availability, it is therefore important to establish the range of performance between various receiver models. In this paper, two performance evaluations are conducted for codeless and semicodeless receivers, under auroral scintillation conditions: 1) a comparison of receiver tracking performance between two adjacent regional networks, and 2) a comparison of three collocated receivers during identical scintillation conditions.

\section{Cycle Slip Detection and Tracking Statistics}

In deriving tracking statistics for the receivers studied in this paper, it was necessary to establish consistent and reliable criteria for cycle slip detection. While warning flags and estimates of measurement quality are included in the raw data formats, such information is derived from internal processing algorithms which are manufacturer-dependent. Raw loss of lock indicators are also not included in receiver-independent (i.e. RINEX) formats. The many users of IGS RINEX data must employ external cycle slip detection algorithms in order to assess the quality of carrier phase data, and such a receiverindependent cycle slip detection algorithm is employed here. This method is based on the robust algorithm developed by Blewitt (1990), and major features of this method are described as follows:

1) An estimate of the widelane bias is derived-by differencing the narrowlane code observable and the widelane phase combination. While this estimate is corrupted by code noise and multipath, discontinuities of one widelane cycle are reliably detected in time series of this observable through comparisons with a threshold value based on observed RMS scatter.

2) In the rare case that identical cycle slips occur simultaneously on L1 and L2, a second detection criterion is required. This criterion is based on the computed differences between the geometry-free carrier phase observable and the smoothed geometry-free code observable, which allows an estimate of the geometry-free bias. This bias should remain constant over time, and a cycle slip is detected when a predetermined discontinuity threshold is exceeded.

3) Once a cycle slip has been detected, it is necessary to establish whether the cycle slip occurred on L1, L2, or both carriers. This technique is based on extrapolation of the ionospheric combination to isolate the L2 bias (using knowledge of the widelane bias). For a highly variable ionosphere this method is appropriate only for identifying the larger cycle slips on L1 or L2. For receivers such as those studied here, however, where cycle slips are consistently larger than 10 cycles (on L1 or L2) and extrapolation intervals are short (5s), this method has proven successful.

In this paper, the receiver tracking performance is quantified in terms of both the number of cycle slips detected and the number of observations missing during reacquisition periods. It is necessary to consider the number of missing observations as an additional statistic in order to accurately reflect the range of receiver performance. For receivers with long reacquisition periods, ten or twenty (or more) observations may be missing during data dropouts following loss of lock. In such cases, only one cycle slip will be detected, but the impact on positioning applications is significant in terms of data dropouts.

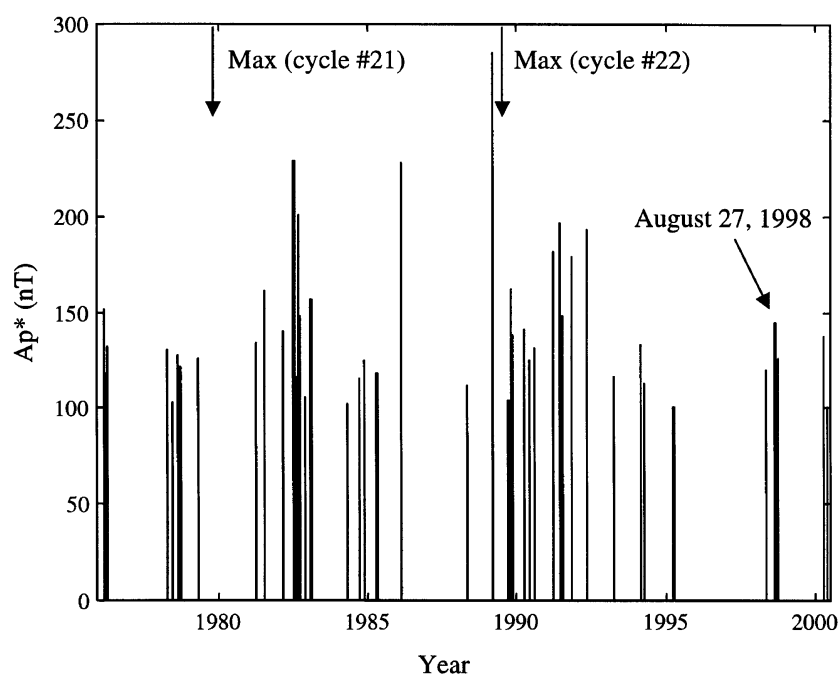

Fig. 1. The Ap* index for severe storm events (AP* $>100)$, during solar cycles $21-23$.

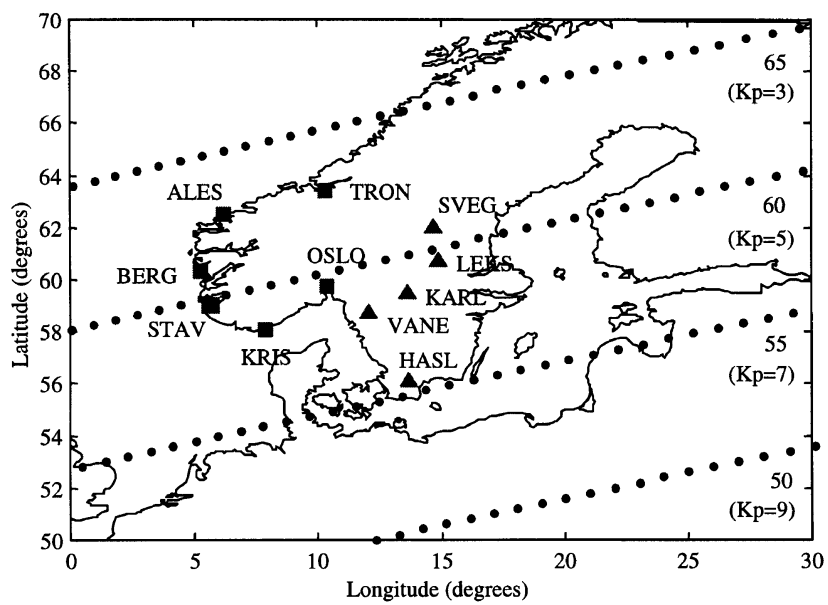

Fig. 2. Geographic locations of stations in the SATREF (square) and SWEPOS (triangle) networks. Corresponding geomagnetic latitudes are also plotted (dotted lines), where the position of the equatorward auroral boundary is indicated for low $(\mathrm{Kp}=3)$, moderate $(\mathrm{Kp}=5)$, major $(\mathrm{Kp}=7)$ and intense $(\mathrm{Kp}=9)$ levels of auroral activity. 

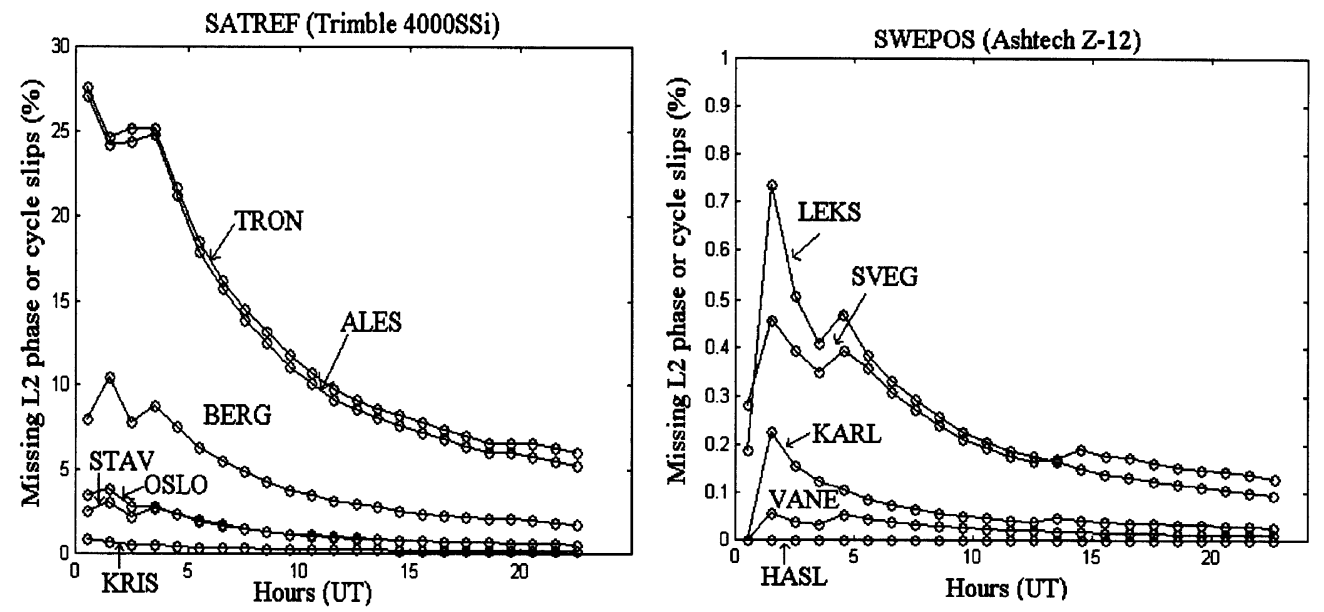

Fig. 3. Percentage of L2 cycle slips and missing phase observations for stations in the SATREF and SWEPOS networks, August $27,1998$.

\section{Substorm Event-August 27, 1998}

A substorm event took place August 27, 1998, during which auroral disturbances were observed in all high latitude local time sectors. The storm effects continued over a 24-hour period, with the strongest effects observed during the interval 0-6 UT. This event was the most severe storm of the present solar cycle (until May 2000), as measured using the global Ap* index from NOAA—see http: //www. ngdc . noaa.gov/stp/GEOMAG/apstar.html for a description of this index. While this event took place several years before solar maximum, peaks in magnetic storm activity tend to occur several years before and after solar maximum. The largest storms are generally observed one to two years after solar maximum. This is illustrated in Fig. 1 for the previous two solar cycles.

During this event, $0.20 \mathrm{~Hz}$ dual frequency observations were available from two regional GPS networks: the SATREF (SATellittbasert REFeransesystem) network in Norway, and the SWEPOS (Swedish Permanent GPS Network) network in Sweden (Fig. 2). The SATREF stations were equipped with Trimble $4000 \mathrm{SSi}$ (codeless) receivers and the SWEPOS stations were equipped with Ashtech Z12 (semicodeless) receivers. While ionospheric effects, at a given time, are not necessarily correlated between the two networks, Kp values (which are a measure of auroral and subauroral ionospheric activity) reached 7-8 during this event. Discrete aurora, as observed in UV images of the oval from the POLAR spacecraft, were present over both networks.

An evaluation of receiver tracking performance at stations in the two networks was performed, where L2 tracking performance was quantified in terms of the hourly percentage of cycle slips and missing observations. Only those observations at elevation angles greater than 15 degrees were considered, in order to eliminate tracking errors due to multipath and lower SNR. Figure 3 illustrates the receiver tracking performance for each station within the two networks. Two observations are made, based on this figure:

1) the receiver tracking performance is degraded more for stations at higher geomagnetic latitudes.

2) the receiver tracking performance is significantly de-

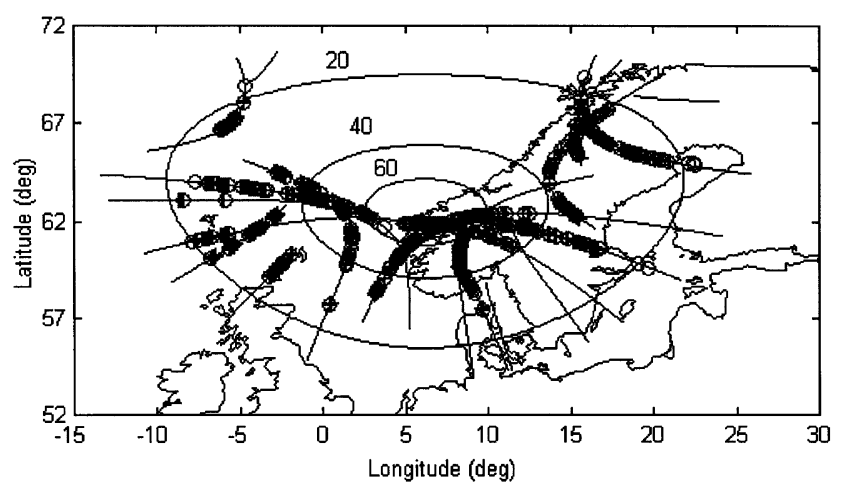

Fig. 4. Satellite-receiver ionospheric pierce points (at altitudes of 350 $\mathrm{km}$ ) for reference station Alesund (ALES), August 27, 1998. Contours for 20, 40 and 60 degree elevations are plotted, in addition to symbols representing L2 cycle slips (circle) and missing L2 phase observations (plus).

graded for receivers within the SATREF network (up to $28 \%$ tracking errors), versus receiver performance within the SWEPOS network (less than 1\% tracking errors-all stations).

Observation 1 is consistent for both networks, and arises from the increased number of observations with lines-ofsight passing through the auroral region at higher latitudes. Observation 2 arises from two effects: an increased number of cycle slip intervals, and the longer reacquisition periods (intervals of missing phase observations), for the Trimble (codeless) receivers. It is important to also note that $\mathrm{L} 2$ code observations were missing during L2 phase reacquisition periods for the Trimble $4000 \mathrm{SSi}$ - this was not the case for the Ashtech Z-12.

The spatial distribution of corrupt observations for Alesund (ALES) is shown in Fig. 4 for the period 0-6 UT. Scintillation effects are observed at geographic latitudes in the range $58^{\circ}-70^{\circ}$ geographic. The equatorward extent of observed tracking errors is consistent with the equatorward oval boundary during a major storm (see Fig. 2). While many tracking errors are present at lower elevation angles $\left(20^{\circ}-\right.$ 
Table 1. Receiver tracking statistics for L2 (0-6 UT, elevation angles $>15^{\circ}$ ).

\begin{tabular}{lcrrr}
\hline Network & $\begin{array}{c}\text { \# cycle slip } \\
\text { intervals }\end{array}$ & $\begin{array}{c}\text { \# L2 phase } \\
\text { missing }\end{array}$ & $\begin{array}{c}\text { \# L2 code } \\
\text { missing }\end{array}$ & $\begin{array}{c}\text { Average reacquisiton } \\
\text { period (s) }\end{array}$ \\
\hline SATREF & $851(22)$ & $13638(7)$ & $13638(7)$ & $80(6)$ \\
SWEPOS & $222(13)$ & $654(0)$ & $0(0)$ & $15(0)$ \\
\hline
\end{tabular}

$40^{\circ}$ ), a significant number of tracking errors are observed at elevation angles of $40^{\circ}-60^{\circ}$ and more. It is possible that tracking errors at higher latitudes (i.e. above $68^{\circ}$ ) are due to patches of irregularities in the polar cap.

Overall results are summarized in Table 1, for each network, where quiet baseline statistics (for the same satellite constellation) are given in brackets. While results from the two networks suggest significant performance differences for the Trimble and Ashtech receivers during periods of high latitude scintillations (L2 tracking), it may be argued that ionospheric conditions were not identical for the two networks, which were located at different local times, and slightly different latitudes. In order to verify the results of this section, an independent test was conducted in which the tracking performance of three different receivers was compared directly, under identical scintillation conditions. This test is described in the next section.

\section{Receiver Performance Comparison-June 28, 1999}

A receiver performance comparison was conducted in the Yukon Territory, Northern Canada, during the period 21-30 June 1999. In this test, a Trimble 4000 SSi (codeless), a NovAtel MiLLennium (semicodeless), and an Ashtech Z-12 (semicodeless) were tested under identical conditions. Antennas for each receiver were mounted on the roof of a stationary vehicle at Haines Junction $\left(60.8^{\circ} \mathrm{N}, 137.5^{\circ} \mathrm{W}\right)$, and data were collected during nighttime hours when aurora are strongest (approximately 1900-0200 local time, 4-11 UT) at a rate of $0.20 \mathrm{~Hz}$. As in Section 3, only observations with elevation angles greater than 15 degrees were considered.

During the test period, irregularities in auroral electron density were observed during a moderate-major geomagnetic storm event $(\mathrm{Kp}=6)$ that took place 6-9 UT on June 28,1999 . Degraded receiver tracking performance was observed predominantly in the period 7-9 UT, consistent with the storm period. It must be noted that this event does not necessarily qualify as a full substorm event, which requires several distinct stages of development (see Skone and Cannon (1998) for a basic description of such events). It can, however, be inferred that elements of enhanced auroral activity are present, as reflected in the TEC variations (Fig. 6).

Figure 5 shows the tracking performance of each receiver, for each satellite, in terms of the observed L2 cycle slips and missing L2 phase observations at each epoch. The overall L2 tracking statistics are given in Table 2, for the three receivers, where quiet baseline statistics (for the same satellite constellation) are given in brackets. L1 tracking performance was not affected significantly during this event. It is observed that all receivers experience degraded tracking performance
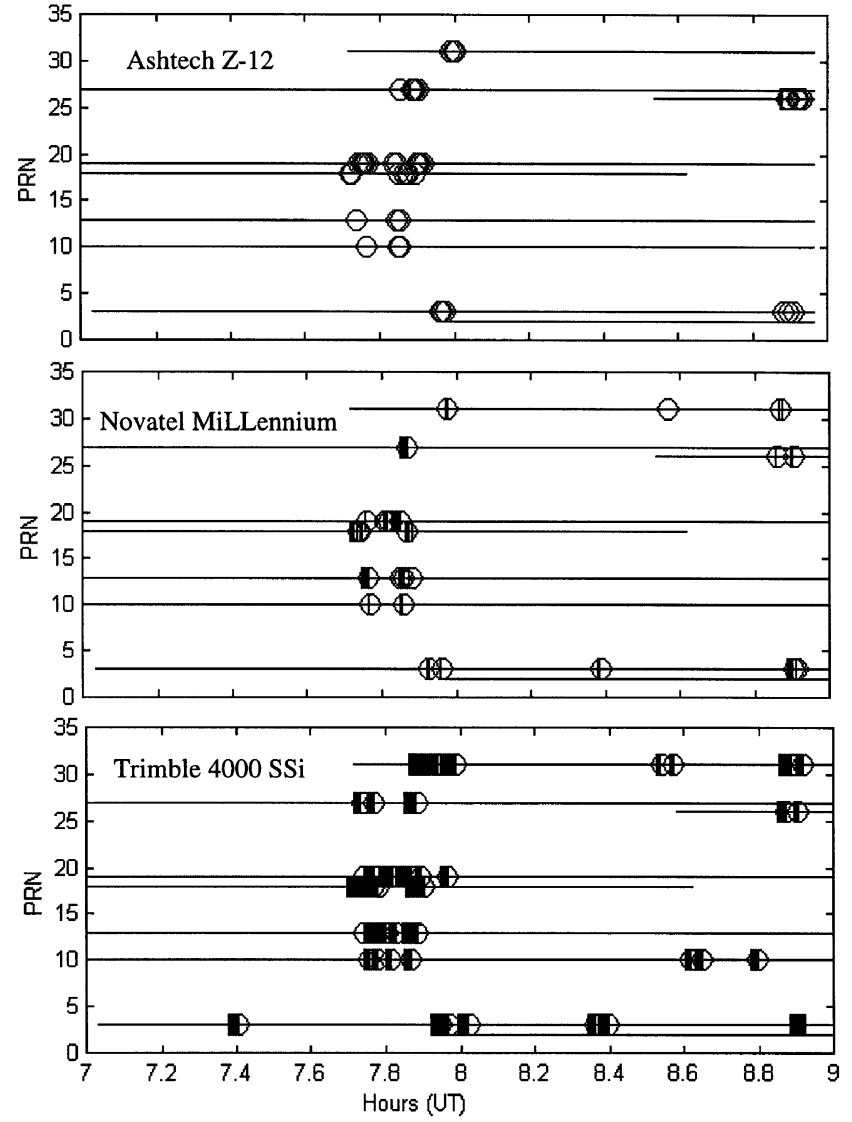

Fig. 5. L2 phase tracking performance for individual satellites tracked by each receiver, June 28, 1999. The symbols denote cycle slips (circle) and missing observations (plus).

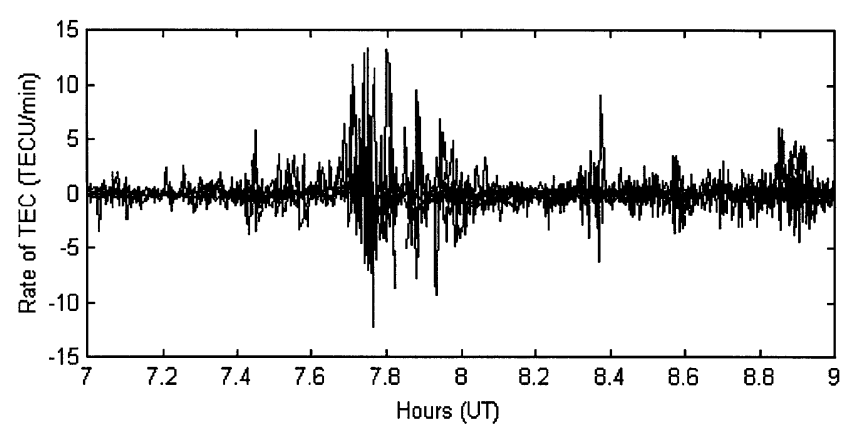

Fig. 6. Rate of TEC (mapped to vertical), as derived using all satellite observations from the Ashtech Z-12 receiver, June 28, 1999, where $1 \mathrm{TECU}=10^{16} \mathrm{el} / \mathrm{m}^{2}$.

predominantly during the 20-minute period 7.7-8 UT and, to a lesser extent, during shorter discrete intervals. For the Trimble receiver, each cycle slip interval includes a long data dropout. Shorter data dropouts are observed for the NovAtel receiver. The corrupt observations occur during periods of enhanced rates of TEC (Fig. 6). The spatial distribution of corrupt observations is shown in Fig. 7, for the Trimble receiver. Corrupt observations are observed over a range of elevation angles, from 15 to 75 degrees. 
Table 2. Receiver tracking statistics for L2 (7-9 UT, elevation angles $>15^{\circ}$ )

\begin{tabular}{lcrrc}
\hline $\begin{array}{l}\text { Receiver } \\
\text { type }\end{array}$ & $\begin{array}{c}\text { \# cycle slip } \\
\text { intervals }\end{array}$ & $\begin{array}{c}\text { \# L2 phase } \\
\text { missing }\end{array}$ & $\begin{array}{c}\text { \# L2 code } \\
\text { missing }\end{array}$ & $\begin{array}{c}\text { Average reacquisiton } \\
\text { period (s) }\end{array}$ \\
\hline Trimble & $40(2)$ & $782(1)$ & $782(1)$ & $100(5)$ \\
NovAtel & $36(2)$ & $103(0)$ & $0(0)$ & $7(0)$ \\
Ashtech & $66(1)$ & $2(0)$ & $0(0)$ & $0(0)$ \\
\hline
\end{tabular}

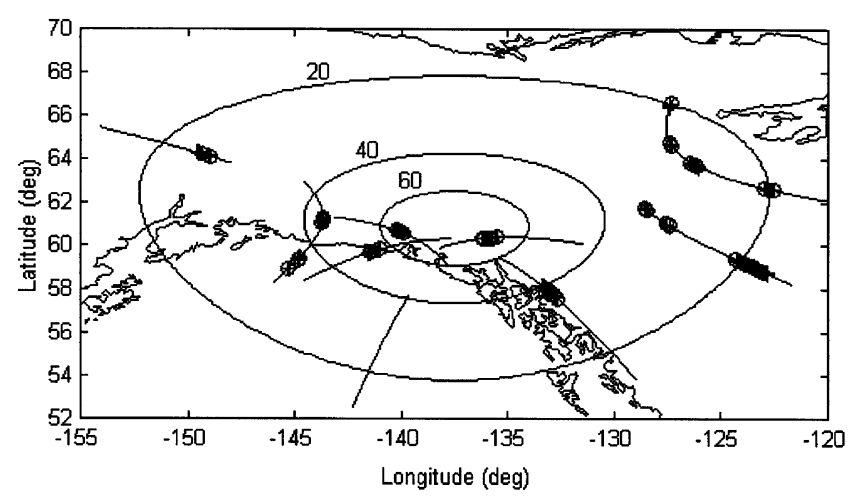

Fig. 7. Satellite-receiver ionospheric pierce points (at altitudes of $350 \mathrm{~km}$ ) for Haines test site, June 28, 1999. Contours for 20, 40 and 60 degree elevations are plotted, in addition to symbols representing L2 cycle slips (circle) and missing L2 phase observations (plus).

\section{Conclusions}

Results of Sections 3 and 4 demonstrate degraded receiver tracking peformance under scintillation conditions. For the Trimble 4000 SSi receiver tested here, up to $28 \%$ of L 2 phase observations were corrupted at high latitude stations during an intense substorm event, compared with less than $1 \%$ for Ashtech Z-12 receivers. Discrepancies in tracking performance are attributed primarily to the longer reacquisition periods observed for the Trimble receiver; L2 code observations were also missing during these reacquisition periods. These results were verified in an independent field test, where the Ashtech Z-12, Trimble 4000 SSi and NovAtel MiLLennium receivers were tested under identical scintillation conditions. In this test the Trimble receiver again experienced extended data dropouts, with additional losses of phase lock during periods when the Ashtech and NovAtel receivers maintained continuous lock on the given satellite. Discrepancies also exist between results for the two semicodeless receivers in Section 4. The NovAtel receiver experienced some short data dropouts, while the Ashtech had only two missing observations.

Differences in receiver performance are a result of many factors-antenna gain patterns, tracking loop bandwidths and internal processing algorithms — and it is difficult to infer the limiting factors for a given receiver. It is likely, however, that degraded performance of the Trimble receiver is due to the codeless tracking technology employed in the L 2 tracking loops, versus the semicodeless technology for the Ashtech and NovAtel receivers. From a user standpoint, there is a range of performance for the various survey-grade receivers, even for those employing similar tracking techniques. Such results suggest the importance of considering not only the magnitude of scintillations, but also the type of GPS receiver used in data collection campaigns, when assessing the impact of ionospheric activity on GPS receiver performance.

Two storm events were studied in this paper, and it is important to note the different magnitudes of these events. The August 27, 1998 event was one of the largest storms to occur in the current solar cycle, and associated scintillations should be considered as approaching the upper bounds of high latitude effects at solar maximum. During this event, scintillations persisted over many hours, significantly degrading receiver tracking performance in the SATREF (codeless receiver) network. Performance of the semicodeless receiver was encouraging, however, as was the negligible effect on L1 tracking performance for both types of receivers. In contrast, the June 28, 1999 event was a moderate storm, with only short-lived scintillation effects observed. These limited periods of scintillation occur more frequently than the intense substorm events, and are more representative of typical high latitude scintillations.

Acknowledgments. The authors thank Dr. D. Schwarz for his assistance in the Yukon data collection. The authors also acknowledge the Norwegian Mapping Authority and the National Survey of Sweden for providing GPS data.

\section{References}

Aarons, J., Global morphology of ionospheric scintillations, Proceedings of the IEEE, 70, no. 4, 1982.

Blewitt, G., An automatic editing algorithm for GPS data, Geophys. Res. Lett., 17(3), 199-202, 1990.

Clynch, J. and C. Henry, Ionospheric effects on GPS and DGPS in polar regions, Proceedings of the ION GPS-94, Salt lake City, Utah, September, 1994.

Clynch, J. and J. Aarons, High latitude GPS observations and receiver constraints, Proceedings of the Ionospheric effects Symposium 1996, edited by J. M. Goodman, 1996.

Hargreaves, J. K., The Solar-Terrestrial Environment, Cambridge University Press, Cambridge, 1992.

Knight, M., M. Cervera, and A. Finn, A comparison of predicted and measured GPS performance in an ionospheric scintillation environment, Proceedings of the ION GPS-99, Nashville, Tennessee, September, 1999.

Leick, A., GPS Satellite Surveying, second edition, JohnWiley \& Sons, U.S.A., 1995.

Nichols, J., A. Hansen, T. Walter, and P. Enge, High latitude measurements of ionospheric scintillation using the NSTB, Proceeedings of the ION National Technical Meeting, San Diego, California, January, 1999.

Rostoker, G., Some observational constraints for substorm models, in Magnetospheric Substorms, edited by J. R. Kan, T. A. Potemra, S. Kokubun, and T. Iijima, pp. 61-72, Geophysical Monograph 64, AGU, Washington, D.C., 1991.

Skone, S. and M. E. Cannon, Detailed analysis of auroral zone WADGPS ionosphere grid accuracies during magnetospheric substorm event, Proceedings of the ION GPS-98, Nashville, Tennessee, September, 1998.

S. Skone (e-mail: sskone@ensu.ucalgary.ca) and M. de Jong 